\title{
The Euler Class in the Simplicial de Rham Complex
}

\author{
Naoya Suzuki \\ (Communicated by Yusuf Yayli)
}

\begin{abstract}
We exhibit a cocycle in the simplicial de Rham complex which represents the Euler class. As an application, we construct a Lie algebra cocycle on $L \mathfrak{s o}(4)$.
\end{abstract}

Keywords: Characteristic classes; simplicial manifold.

AMS Subject Classification (2010): Primary:53-XX.

For any Lie group $G$, we can define a simplicial manifold $\{N G(*)\}$ and a double complex $\Omega^{*}(N G(*))$ on it. In classical theory, it is well-known that the cohomology ring of the total complex $\Omega^{*}(N G)$ is isomorphic to $H^{*}(B G)$ where $B G$ is a classifying space of $G$, which is not a manifold in general [2] [5] [6].

In [4], Dupont introduced another double complex $A^{*, *}(N G)$ on $N G$ such that the cohomology ring of its total complex $A^{*}(N G)$ is also isomorphic to $H^{*}(B G)$. He used it to construct a homomorphism from $I^{*}(G)$, the $G$-invariant polynomial ring over Lie algebra $\mathcal{G}$, to $H^{*}(B G)$. By using Dupont's method, in [8] the author exhibited cocycles in $\Omega^{*}(N G)$ which represent the Chern characters. In this paper, we will exhibit cocycles which represent the Euler classes.

Using a cocycle in $\Omega^{*}(N G)$, we can construct a cocycle in the local truncated complex $\left[\sigma_{<p} \Omega_{\text {loc }}^{*}(N G)\right]$ due to Brylinski [3]. Furthermore, we can obtain a Lie algebra cocycle of a free loop group $L G$. Following Brylinski's idea, we will construct a Lie algebra 2-cocycle on $L \mathfrak{s o}(4)$ using a cocycle in $\Omega^{4}(N S O(4))$.

\section{Review of the universal Chern-Weil Theory}

In this section we recall the universal Chern-Weil theory following [5]. For any Lie group $G$, we have simplicial manifolds $N \bar{G}, N G$ and simplicial $G$-bundle $\gamma: N \bar{G} \rightarrow N G$ as follows:

$$
\begin{aligned}
& N \bar{G}(q)=\overbrace{G \times \cdots \times G}^{q+1-\text { times }} \ni\left(g_{1}, \cdots, g_{q+1}\right) \\
& N G(q)=\overbrace{G \times \cdots \times G}^{\text {q-times }} \ni\left(h_{1}, \cdots, h_{q}\right): \\
& \text { face operators } \varepsilon_{i}: N G(q) \rightarrow N G(q-1) \\
& \varepsilon_{i}\left(h_{1}, \cdots, h_{q}\right)= \begin{cases}\left(h_{2}, \cdots, h_{q}\right) & i=0 \\
\left(h_{1}, \cdots, h_{i} h_{i+1}, \cdots, h_{q}\right) & i=1, \cdots, q-1 \\
\left(h_{1}, \cdots, h_{q-1}\right) & i=q .\end{cases}
\end{aligned}
$$

We define $\gamma: N \bar{G} \rightarrow N G$ as $\gamma\left(g_{0}, \cdots, g_{q}\right)=\left(g_{0} g_{1}^{-1}, \cdots, g_{q-1} g_{q}{ }^{-1}\right)$.

For any simplicial manifold $X=\left\{X_{*}\right\}$, we can associate a topological space $\|X\|$ called the fat realization. It is well-known that $\|\gamma\|$ is the universal bundle $E G \rightarrow B G$ [7].

Now we introduce a double complex associated to a simplicial manifold. 
Definition 1.1. For any simplicial manifold $\left\{X_{*}\right\}$ with face operators $\left\{\varepsilon_{*}\right\}$, we define a double complex as follows:

$$
\Omega^{p, q}(X):=\Omega^{q}\left(X_{p}\right)
$$

Derivatives are:

$$
d^{\prime}:=\sum_{i=0}^{p+1}(-1)^{i} \varepsilon_{i}^{*}, \quad d^{\prime \prime}:=(-1)^{p} \times \text { the exterior differential on } \Omega^{*}\left(X_{p}\right) .
$$

For $N G$ and $N \bar{G}$ the following holds [2] [5] [6].

Theorem 1.1. There exist ring isomorphisms

$$
H\left(\Omega^{*}(N \bar{G})\right) \cong H^{*}(E G), \quad H\left(\Omega^{*}(N G)\right) \cong H^{*}(B G) .
$$

Here $\Omega^{*}(N \bar{G})$ and $\Omega^{*}(N G)$ mean the total complexes.

There is another double complex associated to a simplicial manifold.

Definition 1.2 ([4]). A simplicial $n$-form on a simplicial manifold $\left\{X_{p}\right\}$ is a sequence $\left\{\phi^{(p)}\right\}$ of $n$-forms $\phi^{(p)}$ on $\Delta^{p} \times X_{p}$ such that

$$
\left(\varepsilon^{i} \times i d\right)^{*} \phi^{(p)}=\left(i d \times \varepsilon_{i}\right)^{*} \phi^{(p-1)} \text { on } \Delta^{p-1} \times X_{p} .
$$

Here $\varepsilon^{i}$ is the canonical $i$-th face operator of $\Delta^{p}$.

Let $A^{k, l}(X)$ be the set of all simplicial $(k+l)$-forms on $\Delta^{p} \times X_{p}$ which are expressed locally of the form

$$
\sum a_{i_{1} \cdots i_{k} j_{1} \cdots j_{l}}\left(d t_{i_{1}} \wedge \cdots \wedge d t_{i_{k}} \wedge d x_{j_{1}} \wedge \cdots \wedge d x_{j_{l}}\right)
$$

where $\left(t_{0}, t_{1}, \cdots, t_{p}\right)$ are the barycentric coordinates in $\Delta^{p}$ and $x_{j}$ are the local coordinates in $X_{p}$. We define derivatives as:

$$
\begin{gathered}
d^{\prime}:=\text { the exterior differential on } \Delta^{p} \\
d^{\prime \prime}:=(-1)^{k} \times \text { the exterior differential on } X_{p} .
\end{gathered}
$$

Then $\left(A^{k, l}(X), d^{\prime}, d^{\prime \prime}\right)$ is a double complex and the following theorem holds.

Theorem 1.2 ([4]). Let $A^{*}(X)$ denote the total complex of $A^{*, *}(X)$. A map $I_{\Delta}: A^{*}(X) \rightarrow \Omega^{*}(X)$ defined as $I_{\Delta}(\alpha):=$ $\int_{\Delta^{p}}\left(\left.\alpha\right|_{\Delta^{p} \times X_{p}}\right)$ induces a natural ring isomorphism $I_{\Delta}^{*}: H\left(A^{*}(X)\right) \cong H\left(\Omega^{*}(X)\right)$.

Let $\mathcal{G}$ denote the Lie algebra of $G$. A connection on a simplicial $G$-bundle $\pi:\left\{E_{p}\right\} \rightarrow\left\{M_{p}\right\}$ is a sequence of 1-forms $\{\theta\}$ on $\left\{E_{p}\right\}$ with coefficients $\mathcal{G}$ such that $\theta$ restricted to $\Delta^{p} \times E_{p}$ is a usual connection form.

There is a canonical connection $\theta \in A^{1}(N \bar{G})$ on $\gamma: N \bar{G} \rightarrow N G$ defined as follows:

$$
\left.\theta\right|_{\Delta^{p} \times N \bar{G}(p)}:=t_{0} \theta_{0}+\cdots+t_{p} \theta_{p} .
$$

Here $\theta_{i}$ is defined as $\theta_{i}=\operatorname{pr}_{i}^{*} \bar{\theta}$ where $\operatorname{pr}_{i}: \Delta^{p} \times N \bar{G}(p) \rightarrow G$ is the projection into the $(i+1)$-th factor of $N \bar{G}(p)$ and $\bar{\theta}$ is the Maurer-Cartan form of $G$. We obtain also its curvature $\Omega \in A^{2}(N \bar{G})$ on $\gamma$ as:

$$
\left.\Omega\right|_{\Delta^{p} \times N \bar{G}(p)}=\left.d \theta\right|_{\Delta^{p} \times N \bar{G}(p)}+\frac{1}{2}\left[\left.\theta\right|_{\Delta^{p} \times N \bar{G}(p)},\left.\theta\right|_{\Delta^{p} \times N \bar{G}(p)}\right] .
$$

Let $I^{*}(G)$ denote the ring of $G$-invariant polynomials on $\mathcal{G}$. For $P \in I^{k}(G)$, we restrict $P(\Omega) \in A^{2 k}(N \bar{G})$ to each $\Delta^{p} \times N \bar{G}(p)$ and apply the usual Chern-Weil theory then we have $I_{\Delta}(P(\Omega)) \in \Omega^{2 k}(N G)$. In this way we have a homomorphism I* $(G) \rightarrow H\left(\Omega^{*}(N G)\right)$ which maps $P \in I^{*}(G)$ to $\left[I_{\Delta}(P(\Omega))\right]$. 


\section{The Euler class in the double complex}

In this section we exhibit a cocycle in $\Omega^{*}(N S O(2 p))$ which represents the Euler class of the universal bundle $E S O(2 p) \rightarrow B S O(2 p)$. Throughout this section, $G$ means $S O(2 p)$.

Recall that the polynomial on $\mathfrak{s o}(2 p)$ called Pfaffian is defined as follows:

$$
\operatorname{Pf}(A, \cdots, A)=\frac{1}{2^{2 p} \pi^{p} p !} \sum_{\tau \in \mathfrak{S}_{2 p}} \operatorname{sgn}(\tau) a_{\tau(1) \tau(2)} \cdots a_{\tau(2 p-1) \tau(2 p)} .
$$

Here $a_{i j}$ is a $(i, j)$ entry of $A \in \mathfrak{s o}(2 p)$.

\subsection{The cochain on the edge}

We first give the cochain in $\Omega^{2 p+1}(N \bar{G}(1))$ which corresponds to the Euler class. This is given by integrating $\operatorname{Pf}\left(\left.\Omega\right|_{\Delta^{1} \times N \bar{G}(1)}\right)$ along $\Delta^{1}$. Since $\left.\Omega\right|_{\Delta^{1} \times N \bar{G}(1)}=-d t_{1} \wedge\left(\theta_{0}-\theta_{1}\right)-t_{0} t_{1}\left(\theta_{0}-\theta_{1}\right)^{2}$, we can see $\operatorname{Pf}\left(\left.\Omega\right|_{\Delta^{1} \times N \bar{G}(1)}\right)$ is equal to

$$
\begin{array}{r}
\frac{1}{2^{2 p} \pi^{p} p !} \sum_{\tau \in \mathfrak{S}_{2 m}} \operatorname{sgn}(\tau)\left(\left(-d t_{1} \wedge\left(\theta_{0}-\theta_{1}\right)-t_{0} t_{1}\left(\theta_{0}-\theta_{1}\right)^{2}\right)_{\tau(1) \tau(2)}\right. \\
\left.\cdots\left(-d t_{1} \wedge\left(\theta_{0}-\theta_{1}\right)-t_{0} t_{1}\left(\theta_{0}-\theta_{1}\right)^{2}\right)_{\tau(2 p-1) \tau(2 p)}\right) .
\end{array}
$$

We set:

$$
\begin{aligned}
\bar{P}_{\tau}^{k}:=\left(\theta_{0}-\theta_{1}\right)_{\tau(1) \tau(2)}^{2} & \cdots\left(\theta_{0}-\theta_{1}\right)_{\tau(2 k-3) \tau(2 k-2)}^{2}\left(\theta_{0}-\theta_{1}\right)_{\tau(2 k-1) \tau(2 k)} \\
& \left(\theta_{0}-\theta_{1}\right)_{\tau(2 k+1) \tau(2 k+2)}^{2} \cdots\left(\theta_{0}-\theta_{1}\right)_{\tau(2 p-1) \tau(2 p)}^{2} .
\end{aligned}
$$

Then the following equation holds.

$$
\int_{\Delta^{1}} \operatorname{Pf}\left(\left.\Omega\right|_{\Delta^{1} \times N \bar{G}(1)}\right)=(-1)^{p} \frac{1}{2^{2 p} \pi^{p} p !}\left(\int_{0}^{1}\left(t_{0} t_{1}\right)^{p-1} d t_{1}\right) \sum_{\tau \in \mathfrak{S}_{2 p}} \sum_{k=1}^{p} \operatorname{sgn}(\tau) \bar{P}_{\tau}^{k} .
$$

Now we obtain the cochain in $\Omega^{2 p-1}(N G(1))$.

Proposition 2.1. The cochain $\mu_{p}$ in $\Omega^{2 p-1}(N G(1))$ which corresponds to the Euler class is given as follows:

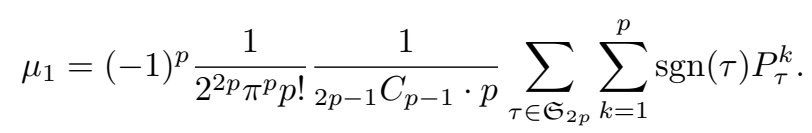

Here $P_{\tau}^{k}$ is defined as:

$$
P_{\tau}^{k}:=\left(h^{-1} d h\right)_{\tau(1) \tau(2)}^{2} \cdots\left(h^{-1} d h\right)_{\tau(2 k-3) \tau(2 k-2)}^{2}\left(h^{-1} d h\right)_{\tau(2 k-1) \tau(2 k)}\left(h^{-1} d h\right)_{\tau(2 k+1) \tau(2 k+2)}^{2} \cdots\left(h^{-1} d h\right)_{\tau(2 p-1) \tau(2 p)}^{2} .
$$

Proof. This follows from the equation $\int_{0}^{1}\left(t_{0} t_{1}\right)^{p-1} d t_{1}=\frac{1}{2 p-1 C_{p-1} \cdot p}$ and $\gamma^{*} \sum_{\tau \in \mathfrak{S}_{2 p}} \operatorname{sgn}(\tau) P_{\tau}^{k}=\sum_{\tau \in \mathfrak{S}_{2 p}} \operatorname{sgn}(\tau) \bar{P}_{\tau}^{k}$.

As a special case of Proposition 3.1, we obtain the following theorem.

Theorem 2.1. In the case of $G=S O(2)$, the cocycle $E_{1,1}$ in $\Omega^{2}(N G)$ which represents the Euler class of $E S O(2) \rightarrow$ $B S O(2)$ is given as follows:

$$
E_{1,1}=\frac{1}{4 \pi}\left(-\left(h^{-1} d h\right)_{12}+\left(h^{-1} d h\right)_{21}\right) \in \Omega^{1}(S O(2)) .
$$

If we write an element $h$ in $S O(2)$ as

$$
h=\left(\begin{array}{cc}
\cos \theta & -\sin \theta \\
\sin \theta & \cos \theta
\end{array}\right)
$$


then the equation

$$
h^{-1} d h=\left(\begin{array}{cc}
0 & -d \theta \\
d \theta & 0
\end{array}\right)
$$

holds, so we obtain

$$
E_{1,1}=\frac{1}{4 \pi}(2 d \theta)=\frac{d \theta}{2 \pi}
$$

2.2. The cochain in $\Omega^{p}(N G(p))$

In $\Omega^{p}(N \bar{G}(p)),\left.\Omega\right|_{\Delta^{p} \times N \bar{G}(p)}$ is equal to $-\sum_{i=1}^{p} d t_{i} \wedge\left(\theta_{0}-\theta_{i}\right)-\sum_{0 \leq i<j \leq p} t_{i} t_{j}\left(\theta_{i}-\theta_{j}\right)^{2}$, so the cochain $\int_{\Delta^{p}} \operatorname{Pf}\left(\left.\Omega\right|_{\Delta^{p} \times N \bar{G}(p)}\right)$ in $\Omega^{p}(N \bar{G}(p))$ which corresponds to the Euler class is given as follows:

$$
\frac{1}{2^{2 p} \pi^{p} p !} \sum_{\tau \in \mathfrak{S}_{2 p}} \operatorname{sgn}(\tau)\left(-\sum_{i=1}^{p} d t_{i} \wedge\left(\theta_{0}-\theta_{i}\right)\right)_{\tau(1) \tau(2)} \cdots\left(-\sum_{i=1}^{p} d t_{i} \wedge\left(\theta_{0}-\theta_{i}\right)\right)_{\tau(2 p-1) \tau(2 p)} .
$$

Now

$$
d t_{i} \wedge\left(\theta_{0}-\theta_{i}\right)=d t_{i} \wedge\left\{\left(\theta_{0}-\theta_{1}\right)+\left(\theta_{1}-\theta_{2}\right)+\cdots+\left(\theta_{i-1}-\theta_{i}\right)\right\}
$$

and for any differential forms $\alpha, \beta, \gamma$ and any integer $0 \leq k, l, x \leq p$, the equation $\alpha \wedge\left(d t_{i} \wedge\left(\theta_{x}-\right.\right.$ $\left.\left.\theta_{x+1}\right)_{\tau(2 k-1) \tau(2 k)}\right) \wedge \beta \wedge\left(d t_{j} \wedge\left(\theta_{x}-\theta_{x+1}\right)_{\tau(2 l-1) \tau(2 l)}\right) \wedge \gamma=-\alpha \wedge\left(d t_{j} \wedge\left(\theta_{x}-\theta_{x+1}\right)_{\tau(2 k-1) \tau(2 k)}\right) \wedge \beta \wedge\left(d t_{i} \wedge\right.$ $\left.\left(\theta_{x}-\theta_{x+1}\right)_{\tau(2 l-1) \tau(2 l)}\right) \wedge \gamma$ holds, so the terms of these forms cancel with each other in $\operatorname{Pf}\left(\left.\Omega\right|_{\Delta^{p} \times N \bar{G}(p)}\right)$.

We set:

$$
\varphi_{s}:=h_{1} \cdots h_{s-1} d h_{s} h_{s}^{-1} \cdots h_{1}^{-1} .
$$

Then we can check that $\gamma^{*} \varphi_{s}=g_{1}\left(\theta_{s-1}-\theta_{s}\right) g_{1}^{-1}$ hence we obtain the following proposition.

Proposition 2.2. The cochain $\mu_{p}$ in $\Omega^{p}(N G(p))$ which corresponds to the Euler class is given as follows:

$$
\mu_{p}=\frac{(-1)^{\frac{p(p+1)}{2}}}{2^{2 p} \pi^{p}(p !)^{2}} \sum_{\sigma \in \mathfrak{S}_{p}} \sum_{\tau \in \mathfrak{S}_{2 p}} \operatorname{sgn}(\tau) \operatorname{sgn}(\sigma)\left(\varphi_{\sigma(1)}\right)_{\tau(1) \tau(2)} \cdots\left(\varphi_{\sigma(p)}\right)_{\tau(2 p-1) \tau(2 p)} .
$$

Using Proposition 3.1 and Proposition 3.2, we obtain the cocycle which represents the Euler class of $E S O(4) \rightarrow B S O(4)$ in $\Omega^{4}(N S O(4))$.

Theorem 2.2. In the case of $G=S O(4)$, the cocycle which represents the Euler class of $E S O(4) \rightarrow B S O(4)$ in $\Omega^{4}(N G)$ is the sum of the following $E_{1,3}$ and $E_{2,2}$ :

$$
\begin{gathered}
0 \\
\uparrow d^{\prime \prime} \\
E_{1,3} \in \Omega^{3}(S O(4)) \stackrel{d^{\prime}}{\longrightarrow} \begin{array}{c}
\Omega^{3}(S O(4) \times S O(4)) \\
\uparrow d^{\prime \prime}
\end{array} \\
E_{1,3}=\frac{1}{192 \pi^{2}} \sum_{\tau \in \mathfrak{S}_{4}} \operatorname{sgn}(\tau)\left(\left(h^{-1} d h\right)_{\tau(1) \tau(2)}\left(h^{-1} d h\right)_{\tau(3) \tau(4)}^{2}\right. \\
\left.+\left(h^{-1} d h\right)_{\tau(1) \tau(2)}^{2}\left(h^{-1} d h\right)_{\tau(3) \tau(4)}\right) \\
E_{2,2}=\frac{-1}{64 \pi^{2}} \sum_{\tau \in \mathfrak{S}_{4}} \operatorname{sgn}(\tau)\left(\left(h_{1}^{-1} d h_{1}\right)_{\tau(1) \tau(2)}\left(d h_{2} h_{2}^{-1}\right)_{\tau(3) \tau(4)}\right. \\
\left.+\left(d h_{2} h_{2}^{-1}\right)_{\tau(1) \tau(2)}\left(h_{1}^{-1} d h_{1}\right)_{\tau(3) \tau(4)}\right) .
\end{gathered}
$$




\subsection{The cocycle in $\Omega^{p+q}(N G(p-q))$}

Repeating the same argument in section 3.2, we obtain a cocycle in $\Omega^{p+q}(N G(p-q))$.

We set:

$$
R_{i j}:=\left(\varphi_{i}+\varphi_{i+1}+\cdots+\varphi_{j-1}\right)^{2} \quad(1 \leq i<j \leq p-q+1) .
$$

Theorem 2.3. The cocycle in $\Omega^{p+q}(N G(p-q))(0 \leq q \leq p-1)$ which represents the Euler class of $E S O(2 p) \rightarrow$ $B S O(2 p)$ is

$$
\begin{aligned}
\sum_{\sigma \in \mathfrak{S}_{p-q}, \tau \in \mathfrak{S}_{2 p}} \sum\left(T_{p, q}^{\tau, \sigma}\left(R_{i_{1} j_{1}}\right)_{\tau(1) \tau(2)}\left(\varphi_{\sigma(1)}\right)_{\tau(3) \tau(4)}\right. \\
\left.\cdots\left(R_{i_{q} j_{q}}\right)_{\tau(2 p-3) \tau(2 p-2)}\left(\varphi_{\sigma(p-q)}\right)_{\tau(2 p-1) \tau(2 p)}\right)
\end{aligned}
$$

where $R_{i j}(1 \leq i<j \leq p-q+1)$ are put $q$-times between $\varphi_{\sigma(l)}$ and $\varphi_{\sigma(l+1)}$ or the edge in $\varphi_{\sigma(1)} \cdots \varphi_{\sigma(p-q)}$ permitting overlaps and $\sum$ means the sum of all such forms. $T_{p, q}^{\tau, \sigma}$ is defined as:

$$
T_{p, q}^{\tau, \sigma}=\operatorname{sgn}(\tau) \operatorname{sgn}(\sigma) \frac{(-1)^{p+\frac{(p-q)(p-q-1)}{2}}}{2^{2 p} \pi^{p} p !}\left(\int_{\Delta^{p-q}} \prod_{i<j}\left(t_{i-1} t_{j-1}\right)^{r_{i j}} d t_{1} \wedge \cdots \wedge d t_{p-q}\right)
$$

where $r_{i j}$ means the number of $R_{i j}$ in each form.

Theorem 2.4. In the case of $G=S O(6)$, the cocycle which represents the Euler class in $\Omega^{6}(N G)$ is the sum of the following $E_{1,5}, E_{2,4}$ and $E_{3,3}$ :

$$
\begin{aligned}
& 0 \\
& \uparrow d^{\prime \prime} \\
& E_{1,5} \in \Omega^{5}(G) \stackrel{d^{\prime}}{\longrightarrow} \quad \Omega^{5}(N G(2)) \\
& \uparrow d^{\prime \prime} \\
& E_{2,4} \in \Omega^{4}(N G(2)) \stackrel{d^{\prime}}{\longrightarrow} \quad \Omega^{4}(N G(3)) \\
& \uparrow d^{\prime \prime} \\
& E_{3,3} \in \Omega^{3}(N G(3)) \stackrel{d^{\prime}}{\longrightarrow} 0 \\
& E_{1,5}=\frac{-1}{2^{6} \cdot 180 \pi^{3}} \sum_{\tau \in \mathfrak{S}_{6}} \operatorname{sgn}(\tau)\left(\left(h^{-1} d h\right)_{\tau(1) \tau(2)}^{2}\left(h^{-1} d h\right)_{\tau(3) \tau(4)}\left(h^{-1} d h\right)_{\tau(5) \tau(6)}\right. \\
& +\left(h^{-1} d h\right)_{\tau(1) \tau(2)}\left(h^{-1} d h\right)_{\tau(3) \tau(4)}^{2}\left(h^{-1} d h\right)_{\tau(5) \tau(6)} \\
& \left.+\left(h^{-1} d h\right)_{\tau(1) \tau(2)}\left(h^{-1} d h\right)_{\tau(3) \tau(4)}\left(h^{-1} d h\right)_{\tau(5) \tau(6)}^{2}\right) \\
& E_{2,4}=\frac{1}{2^{6} \cdot 6 \cdot 4 ! \pi^{3}} \sum_{\tau \in \mathfrak{S}_{6}} \operatorname{sgn}(\tau) \\
& \left(\left(h_{1}^{-1} d h_{1}\right)_{\tau(1) \tau(2)}\left(d h_{2} h_{2}^{-1}\right)_{\tau(3) \tau(4)} .\right. \\
& \left(2 h_{1}^{-1} d h_{1} h_{1}^{-1} d h_{1}+2 d h_{2} h_{2}^{-1} d h_{2} h_{2}^{-1}+h_{1}^{-1} d h_{1} d h_{2} h_{2}^{-1}+d h_{2} h_{2}^{-1} h_{1}^{-1} d h_{1}\right)_{\tau(5) \tau(6)} \\
& +\left(h_{1}^{-1} d h_{1}\right)_{\tau(1) \tau(2)}\left(2 h_{1}^{-1} d h_{1} h_{1}^{-1} d h_{1}+2 d h_{2} h_{2}^{-1} d h_{2} h_{2}^{-1}\right. \\
& \left.+h_{1}^{-1} d h_{1} d h_{2} h_{2}^{-1}+d h_{2} h_{2}^{-1} h_{1}^{-1} d h_{1}\right)_{\tau(3) \tau(4)}\left(d h_{2} h_{2}^{-1}\right)_{\tau(5) \tau(6)} \\
& +\left(2 h_{1}^{-1} d h_{1} h_{1}^{-1} d h_{1}+2 d h_{2} h_{2}^{-1} d h_{2} h_{2}^{-1}+h_{1}^{-1} d h_{1} d h_{2} h_{2}^{-1}+d h_{2} h_{2}^{-1} h_{1}^{-1} d h_{1}\right)_{\tau(1) \tau(2)} \\
& \cdot\left(h_{1}^{-1} d h_{1}\right)_{\tau(3) \tau(4)}\left(d h_{2} h_{2}^{-1}\right)_{\tau(5) \tau(6)}
\end{aligned}
$$




$$
\begin{gathered}
-\left(d h_{2} h_{2}^{-1}\right)_{\tau(1) \tau(2)}\left(h_{1}^{-1} d h_{1}\right)_{\tau(3) \tau(4)} \cdot \\
\left(2 h_{1}^{-1} d h_{1} h_{1}^{-1} d h_{1}+2 d h_{2} h_{2}^{-1} d h_{2} h_{2}^{-1}+h_{1}^{-1} d h_{1} d h_{2} h_{2}^{-1}+d h_{2} h_{2}^{-1} h_{1}^{-1} d h_{1}\right)_{\tau(5) \tau(6)} \\
-\left(d h_{2} h_{2}^{-1}\right)_{\tau(1) \tau(2)}\left(2 h_{1}^{-1} d h_{1} h_{1}^{-1} d h_{1}+2 d h_{2} h_{2}^{-1} d h_{2} h_{2}^{-1}\right. \\
\left.+h_{1}^{-1} d h_{1} d h_{2} h_{2}^{-1}+d h_{2} h_{2}^{-1} h_{1}^{-1} d h_{1}\right)_{\tau(3) \tau(4)}\left(h_{1}^{-1} d h_{1}\right)_{\tau(5) \tau(6)} \\
-\left(2 h_{1}^{-1} d h_{1} h_{1}^{-1} d h_{1}+2 d h_{2} h_{2}^{-1} d h_{2} h_{2}^{-1}+h_{1}^{-1} d h_{1} d h_{2} h_{2}^{-1}+d h_{2} h_{2}^{-1} h_{1}^{-1} d h_{1}\right)_{\tau(1) \tau(2)} \\
\left.\cdot\left(d h_{2} h_{2}^{-1}\right)_{\tau(3) \tau(4)}\left(h_{1}^{-1} d h_{1}\right)_{\tau(5) \tau(6)}\right) . \\
\quad\left(h_{1}^{-1} d h_{1}\right)_{\tau(1) \tau(2)}\left(d h_{2} h_{2}^{-1}\right)_{\tau(3) \tau(4)}\left(h_{2} d h_{3} h_{3}^{-1} h_{2}^{-1}\right)_{\tau(5) \tau(6)} \\
-\left(d h_{2} h_{2}^{-1}\right)_{\tau(1) \tau(2)}\left(h_{1}^{-1} d h_{1}\right)_{\tau(3) \tau(4)}\left(h_{2} d h_{3} h_{3}^{-1} h_{2}^{-1}\right)_{\tau(5) \tau(6)} \\
-\left(h_{1}^{-1} d h_{1}\right)_{\tau(1) \tau(2)}\left(h_{2} d h_{3} h_{3}^{-1} h_{2}^{-1}\right)_{\tau(3) \tau(4)}\left(d h_{2} h_{2}^{-1}\right)_{\tau(5) \tau(6)} \\
+\left(h_{2} d h_{3} h_{3}^{-1} h_{2}^{-1}\right)_{\tau(1) \tau(2)}\left(h_{1}^{-1} d h_{1}\right)_{\tau(3) \tau(4)}\left(d h_{2} h_{2}^{-1}\right)_{\tau(5) \tau(6)} \\
+\left(d h_{2} h_{2}^{-1}\right)_{\tau(1) \tau(2)}\left(h_{2} d h_{3} h_{3}^{-1} h_{2}^{-1}\right)_{\tau(3) \tau(4)}\left(h_{1}^{-1} d h_{1}\right)_{\tau(5) \tau(6)} \\
\left.-\left(h_{2} d h_{3} h_{3}^{-1} h_{2}^{-1}\right)_{\tau(1) \tau(2)}\left(d h_{2} h_{2}^{-1}\right)_{\tau(3) \tau(4)}\left(h_{1}^{-1} d h_{1}\right)_{\tau(5) \tau(6)}\right) .
\end{gathered}
$$

\section{The cocycle in a local truncated complex}

We recall the filtered local simplicial de Rham complex due to Brylinski [3].

Definition 3.1 ([3]). The filtered local simplicial de Rham complex $F^{p} \Omega_{\mathrm{loc}}^{*, *}(N G)$ over a simplicial manifold $N G$ is defined as follows:

$$
F^{p} \Omega_{\mathrm{loc}}^{r, s}(N G)= \begin{cases}\lim _{1 \in V \subset G^{r}} \Omega^{s}(V) & \text { if } s \geq p \\ 0 & \text { otherwise. }\end{cases}
$$

Let $F^{p} \Omega^{*}(N G)$ be a filtered complex

$$
F^{p} \Omega^{r, s}(N G)= \begin{cases}\Omega^{s}(N G(r)) & \text { if } s \geq p \\ 0 & \text { otherwise }\end{cases}
$$

and $\left[\sigma_{<p} \Omega^{*}(N G)\right]$ a truncated complex

$$
\left[\sigma_{<p} \Omega^{r, s}(N G)\right]= \begin{cases}0 & \text { if } s \geq p \\ \Omega^{s}(N G(r)) & \text { otherwise. }\end{cases}
$$

Then there is an exact sequence:

$$
0 \rightarrow F^{p} \Omega^{*}(N G) \rightarrow \Omega^{*}(N G) \rightarrow\left[\sigma_{<p} \Omega^{*}(N G)\right] \rightarrow 0
$$

which induces a boundary map $\beta: H^{l}\left(N G,\left[\sigma_{<p} \Omega_{\mathrm{loc}}^{*}\right]\right) \rightarrow H^{l+1}\left(N G,\left[F^{p} \Omega_{\mathrm{loc}}^{*}\right]\right)$.

Let $\mu_{1}+\cdots+\mu_{p}, \mu_{p-q} \in \Omega^{p+q}(N G(p-q))$ be a cocycle in $\Omega^{2 p}(N G)$. Using this cocycle, we can construct a cocycle $\eta$ in $\left[\sigma_{<p} \Omega_{\mathrm{loc}}^{*}(N G)\right]$ in the following way. 
We take a contractible open set $U \subset G$ containing 1 . Using the same argument in [5], we can construct mappings $\left\{\sigma_{l}: \Delta^{l} \times U^{l} \rightarrow U\right\}_{0 \leq l}$ inductively with the following properties:

(1) $\sigma_{0}(p t)=1$;

(2)

$$
\sigma_{l}\left(\varepsilon^{j}\left(t_{0}, \cdots, t_{l-1}\right) ; h_{1}, \cdots, h_{l}\right)= \begin{cases}\sigma_{l-1}\left(t_{0}, \cdots, t_{l-1} ; \varepsilon_{j}\left(h_{1}, \cdots, h_{l}\right)\right) & \text { if } j \geq 1 \\ h_{1} \cdot \sigma_{l-1}\left(t_{0}, \cdots, t_{l-1} ; h_{2}, \cdots, h_{l}\right) & \text { if } j=0 .\end{cases}
$$

We define mappings $\left\{f_{m, q}: \Delta^{q} \times U^{m+q-1} \rightarrow G^{m}\right\}$ as

$$
f_{m, q}\left(t_{0}, \cdots, t_{q} ; h_{1}, \cdots, h_{m+q-1}\right):=\left(h_{1}, \cdots, h_{m-1}, \sigma_{q}\left(t_{0}, \cdots, t_{q} ; h_{m}, \cdots, h_{m+q-1}\right)\right) .
$$

A $(2 p-m-q)$-form $\beta_{m, q}$ on $U^{m+q-1}$ is defined as $\beta_{m, q}=(-1)^{m} \int_{\Delta_{q}} f_{m, q}^{*} \mu_{m}$. Then we define the cochain $\eta$ as the sum of following $\eta_{l}$ on $U^{2 p-1-l}$ for $0 \leq l \leq p-1$ :

$$
\eta_{l}:=\sum_{m+q=2 p-l, p \geq m \geq 1} \beta_{m, q} .
$$

Theorem 3.1 ([3][8]). $\eta:=\eta_{0}+\cdots+\eta_{p-1}$ is a cocycle in $\left[\sigma_{<p} \Omega_{\mathrm{loc}}^{*}(N G)\right]$ whose cohomology class is mapped to $\left[\mu_{1}+\cdots+\mu_{p}\right]$ in $H^{2 p}\left(N G,\left[F^{p} \Omega_{\mathrm{loc}}^{*}\right]\right)$ by a boundary map $\beta: H^{2 p-1}\left(N G,\left[\sigma_{<p} \Omega_{\mathrm{loc}}^{*}\right]\right) \rightarrow H^{2 p}\left(N G,\left[F^{p} \Omega_{\mathrm{loc}}^{*}\right]\right)$.

Proof. See [8].

\section{Construction of a Lie algebra cocycle}

For any Lie group $G$, let $C_{l o c}^{\infty}\left(G^{p}, \mathbb{R}\right)$ denote the group of germs at $(1, \cdots, 1)$ of smooth functions $G^{p} \rightarrow \mathbb{R}$ and $H_{l o c}^{p}(G, \mathbb{R})$ denote the cohomology group of the following complex:

$$
\cdots \rightarrow C_{l o c}^{\infty}\left(G^{p}, \mathbb{R}\right) \stackrel{\delta:=\sum_{i=0}^{p+1}(-1)^{i} \varepsilon_{i}^{*}}{\longrightarrow} C_{l o c}^{\infty}\left(G^{p+1}, \mathbb{R}\right) \rightarrow \cdots
$$

Brylinski constructed a natural cochain $\operatorname{map} \phi: C_{l o c}^{p}(G, \mathbb{R}) \rightarrow C^{p}(\mathcal{G}, \mathbb{R})$ as follows:

$$
\begin{gathered}
\phi(c)\left(\xi_{1}, \cdots, \xi_{p}\right):= \\
{\left[\frac{\partial^{p}}{\partial y_{1} \cdots \partial y_{p}} \sum_{\rho \in \mathfrak{S}_{p}} \operatorname{sgn}(\rho) c\left(\exp \left(y_{\rho(1)} \xi_{\rho(1)}\right), \cdots, \exp \left(y_{\rho(p)} \xi_{\rho(p)}\right)\right)\right]_{y_{i}=0}}
\end{gathered}
$$

where $C^{p}(\mathcal{G}, \mathbb{R})$ is the space of smooth alternating multilinear maps $\mathcal{G} \rightarrow \mathbb{R}$ and $\xi_{i} \in \mathcal{G}$. For example, if we take $\delta c \in C_{l o c}^{\infty}\left(G^{2}, \mathbb{R}\right)$ and set $X_{\rho(i)}:=\exp \left(y_{\rho(i)} \xi_{\rho(i)}\right)$ then

$$
\begin{gathered}
\phi(\delta c)\left(\xi_{1}, \xi_{2}\right)=\left[\frac{\partial^{2}}{\partial y_{1} \partial y_{2}} \sum_{\rho \in \mathfrak{S}_{2}} \operatorname{sgn}(\rho)(\delta c)\left(X_{\rho(1)}, X_{\rho(2)}\right)\right]_{y_{i}=0} \\
=\left[\frac{\partial^{2}}{\partial y_{1} \partial y_{2}} \sum_{\rho \in \mathfrak{S}_{2}} \operatorname{sgn}(\rho)\left(c\left(X_{\rho(2)}\right)-c\left(X_{\rho(1)} X_{\rho(2)}\right)+c\left(X_{\rho(1)}\right)\right)\right]_{y_{i}=0} \\
=\left[\frac{\partial^{2}}{\partial y_{1} \partial y_{2}}\left(-c\left(X_{1} X_{2}-X_{2} X_{1}\right)\right)\right]_{y_{i}=0}=(d(\phi(c)))\left(\xi_{1}, \xi_{2}\right) .
\end{gathered}
$$

Let $L U$ be the free loop space of a contractible open set $U \subset S O(4)$ containing 1 and ev : $L U \times S^{1} \rightarrow U$ be the evaluation map, i.e. for $\gamma \in L U$ and $\theta \in S^{1}, \operatorname{ev}(\gamma, \theta)$ is defined as $\gamma(\theta)$. Then $\int_{S^{1}}$ ev$^{*}$ maps $\eta_{1} \in \Omega^{1}\left(U^{2}\right)$ to a cochain in $\Omega^{0}\left(L U^{2}\right)$. This cochain defines a cohomology class in local cohomology group $H_{\text {loc }}^{2}(L S O(4), \mathbb{R})$. So as an application of Theorem 3.2, we can obtain a cocycle in $\phi\left(\int_{S^{1}} \mathrm{ev}^{*} \eta_{1}\right) \in C^{2}(L \mathfrak{s o}(4), \mathbb{R})$.

Now we compute this cocycle. We define:

$$
a:=\int_{S^{1}} \mathrm{ev}^{*} \int_{\Delta^{2}} f_{1,2}^{*} E_{1,3}, \quad b:=\int_{S^{1}} \mathrm{ev}^{*} \int_{\Delta^{1}} f_{2,1}^{*} E_{2,2}, \quad c:=\int_{S^{1}} \mathrm{ev}^{*} \eta_{1}
$$


then $c\left(\gamma_{1}, \gamma_{2}\right)=a\left(\gamma_{1}, \gamma_{2}\right)+b\left(\gamma_{1}, \gamma_{2}\right)$ for $\gamma_{1}, \gamma_{2} \in L U$. Recall that

$$
\begin{aligned}
& f_{1,2}\left(t_{0}, t_{1}, t_{2} ; \gamma_{1}(\theta), \gamma_{2}(\theta)\right)=\sigma_{2}\left(t_{0}, t_{1}, t_{2} ; \gamma_{1}(\theta), \gamma_{2}(\theta)\right) \\
& f_{2,1}\left(t_{0}, t_{1}, t_{2} ; \gamma_{1}(\theta), \gamma_{2}(\theta)\right)=\left(\gamma_{1}(\theta), \sigma_{1}\left(t_{0}, t_{1} ; \gamma_{2}(\theta)\right)\right) .
\end{aligned}
$$

In this case we can take:

$$
\begin{gathered}
\gamma_{i}(\theta)=\exp \left(y_{i} \xi_{i}(\theta)\right) \\
\sigma_{1}\left(t_{0}, t_{1} ; \exp \left(y_{2} \xi_{2}(\theta)\right)\right):=\exp \left(t_{1} y_{2} \xi_{2}(\theta)\right) \\
\sigma_{2}\left(t_{0}, t_{1}, t_{2} ; \exp \left(y_{1} \xi_{1}(\theta)\right), \exp \left(y_{2} \xi_{2}(\theta)\right)\right):=\exp \left(\left(1-t_{0}\right) y_{1} \xi_{1}(\theta)\right) \exp \left(t_{2} y_{2} \xi_{2}(\theta)\right)
\end{gathered}
$$

where $\xi_{i} \in L \mathfrak{s o}(4)$. By observing the coefficient of $y_{1} y_{2}$, we see $\phi\left(a\left(\gamma_{1}, \gamma_{2}\right)\right)=0$.

We define a map $\beta_{\gamma_{1}, \gamma_{2}}: S^{1} \times \Delta^{1} \rightarrow S O(4) \times S O(4)$ as follows:

$$
\beta_{\gamma_{1}, \gamma_{2}}\left(\theta ; t_{0}, t_{1}\right):=\left(\gamma_{1}(\theta), \sigma_{1}\left(t_{0}, t_{1} ; \gamma_{2}(\theta)\right)\right) .
$$

Then $b\left(\gamma_{1}, \gamma_{2}\right)=\int_{S^{1} \times \Delta^{1}} \beta_{\gamma_{1}, \gamma_{2}}^{*} E_{2,2}$ and up to $O\left(\left|y_{1}\right|^{2}\right)$ and $O\left(\left|y_{2}\right|^{2}\right)$,

$$
\frac{\partial \beta_{\gamma_{1}, \gamma_{2}}}{\partial \theta}=\left(y_{1} \frac{\partial \xi_{1}(\theta)}{\partial \theta}, t_{1} y_{2} \frac{\partial \xi_{2}(\theta)}{\partial \theta}\right), \quad \frac{\partial \beta_{\gamma_{1}, \gamma_{2}}}{\partial t_{1}}=\left(0, y_{2} \xi_{2}(\theta)\right)
$$

Therefore

$$
\left[\frac{\partial^{2}}{\partial y_{1} \partial y_{2}} b\left(\gamma_{1}, \gamma_{2}\right)\right]_{y_{i}=0}=\frac{-1}{128 \pi^{2}} \sum_{\tau \in \mathfrak{S}_{4}} \operatorname{sgn}(\tau) \int_{0}^{1}\left(\frac{\partial \xi_{1}(\theta)}{\partial \theta}\right)_{\tau(1) \tau(2)} \xi_{2}(\theta)_{\tau(3) \tau(4)} d \theta
$$

Now we obtain the following theorem.

Theorem 4.1. There exists a Lie algebra 2-cocycle $\alpha$ on $L \mathfrak{s o}(4)$ which is expressed as follows:

$$
\begin{gathered}
\alpha\left(\xi_{1}, \xi_{2}\right):=\frac{-1}{128 \pi^{2}} \sum_{\tau \in \mathfrak{S}_{4}}(\operatorname{sgn}(\tau) . \\
\left.\int_{0}^{1}\left(\left(\frac{\partial \xi_{1}(\theta)}{\partial \theta}\right)_{\tau(1) \tau(2)} \xi_{2}(\theta)_{\tau(3) \tau(4)}-\left(\frac{\partial \xi_{2}(\theta)}{\partial \theta}\right)_{\tau(1) \tau(2)} \xi_{1}(\theta)_{\tau(3) \tau(4)}\right) d \theta\right) .
\end{gathered}
$$

\section{References}

[1] Bott, R., On the Chern-Weil homomorphism and the continuous cohomology of the Lie group. Adv. in Math. 11 (1973), $289-303$.

[2] Bott, R., Shulman, H. and Stasheff, J., On the de Rham Theory of Certain Classifying Spaces. Adv. in Math. 20 (1976), 43-56.

[3] Brylinski, J-L., Differentiable cohomology of gauge groups, math.DG/0011069.

[4] Dupont, J.L., Simplicial de Rham cohomology and characteristic classes of flat bundles. Topology Vol 15(1976),233-245, Perg Press.

[5] Dupont, J.L., Curvature and Characteristic Classes, Lecture Notes in Math. 640, Springer Verlag, 1978.

[6] Mostow, M. and Perchick, J., Notes on Gel'fand-Fuks Cohomology and Characteristic Classes (Lectures by Bott). In Eleventh Holiday Symposium. New Mexico State University, December 1973.

[7] Segal, G., Classifying spaces and spectral sequences. Inst. Hautes Études Sci. Publ. Math. No.34 1968 105-112.

[8] Suzuki, N., The Chern character in the Simplicial de Rham Complex. Nihonkai Mathematical Journal Vol.26 (2015), No1, pp.1-13.

\section{Affiliations}

\section{NAOYA SUZUKI}

AdDress: National Institute of the Technology, Akita College, 1-1, Iijima Bunkyo-cho, Akita-shi, Akita-ken, Japan.

E-MAIL: nysuzuki@akita-nct.ac.jp 